\title{
Human Comfort Instrument Design Based on Embedded
}

\author{
Shucheng Chen, Jing Shi*, Xiaobo Li, Ming Cui, Lianwei Su \\ Tianjin Meteorological Observation Centre, Tianjin, China \\ Email: ^Jacque_yuer@126.com
}

How to cite this paper: Chen, S. C., Shi, J., Li, X. B., Cui, M., \& Su, L. W. (2019). Human Comfort Instrument Design Based on Embedded. Journal of Geoscience and Environment Protection, 7, 115-124. https://doi.org/10.4236/gep.2019.76010

Received: November 26, 2018

Accepted: June 24, 2019

Published: June 27, 2019

Copyright $\odot 2019$ by author(s) and Scientific Research Publishing Inc. This work is licensed under the Creative Commons Attribution International License (CC BY 4.0).

http://creativecommons.org/licenses/by/4.0/

(c) (i) Open Access

\begin{abstract}
The traditional human comfort meter has the following defects: the interface is not uniform; the operation is cumbersome and complicated; the interface is unfriendly, and the stability and adaptability are poor. This paper presents a design scheme for human comfort instrument based on embedded system, using S3C2440 embedded development board and the sensors to collect the real-time temperature, relative humidity and wind speed data and to process the collecting data; then obtaining the human body comfort value according to the basic algorithm of human body comfort instrument; giving the human comfort conclusion according to the diastolic index range of human comfort, and showing the temperature and humidity, wind speed, comfort value and conclusion through writing the Qt graphical user interface program. At the same time, the human comfort instrument has the data storage function. The human comfort instrument is high in integration, strong in real time, high in sensitivity, stable and reliable, and it meets the development goals of the intelligent meteorological service, and meets the demand of the meteorological service that is closer to life, and it has broad development prospect.
\end{abstract}

\section{Keywords}

Human Comfort Instrument, Embedded, S3C2440, Qt

\section{Introduction}

Human comfort is a comprehensive reflection of the human body's meteorological elements such as temperature, relative humidity and wind speed, and is a requirement for higher quality meteorological services. The human comfort index is a meteorological indicator defined by the heat exchange between the human and the meteorological environment from the meteorological point of view to evaluate the comfort of the human body in different meteorological environ- 
ments (Moustris et al., 2018). Foreign experts and scholars have laid a good foundation for the study of human comfort. Takaya et al. (2017) proposed realistic temperature based on the cold and hot feelings of the human body under different meteorological conditions; Wallace et al. (2017) studied and proposed the concept of discomfort index, according to the study by the National Weather Service. Regarding forecast summer comfort and working hours; Mostafavi Tehrani et al. (2017) proposed the sensible temperature theory, he considered the heat exchange in the meteorological environment, and calculated the somatosensory temperature model. Domestic experts and scholars started late on the study of human comfort. Slater et al. (2017) proposed the "Climate comfort evaluation model"; Zhu et al. (2014) analyzed the spatial and temporal distribution trend of Hulunbeier human comfort index.

The physiological functions of the human body are affected by various meteorological factors (Li et al., 2016), such as temperature, relative humidity, air pressure, duration of illumination, wind speed and wind direction. Relevant research shows that the three meteorological elements of temperature, relative humidity and wind speed have the greatest impact on human comfort. The human comfort index is a nonlinear equation composed of these three meteorological elements. The equation is derived from the Beijing Meteorological Bureau since 1997. In 2013, the Meteorological Department of Guangdong Province of China developed a "Bio Comfort Measuring Instrument" that reflects the human comfort index (Kai et al., 2014). The comfort measuring instrument consists of sensors, digital collectors, fully automatic water supply systems, and power supply systems. Guangdong Province has begun pilot deployment, and plans to cover 80 to 90 bio-comfort measuring instruments covering the whole province. This "bio-comfort measuring instrument", which reflects the human comfort index, is the first in the country of the scientific research team of the Guangdong Provincial Meteorological Bureau. It has been piloted in Guangzhou and Shenzhen in 2013. As a highlight of Guangdong's pilot project for the construction of meteorological modernization pilots and people's livelihood services, the provincial meteorological department plans to deploy such measuring instruments in the observation fields of cities and counties throughout the province in 2014, initially forming 80 to 90 sites, covering the comfort of the province. The monitoring network will further refine the layout to more hot spots of public concern. Regarding the human comfort index prediction model used (Peng et al., 2011), the human comfort meter designed in this paper is to use the model as the basic algorithm.

The IEEE defines an embedded system as a device for controlling, monitoring, or assisting in the operation of machines and equipment (Wang et al., 2015; Chen et al., 2013). Embedded systems consist of embedded processors, operating systems, application software, and peripherals. The embedded system features real-time, tailorable, unified interface, simple operation, friendly graphical interface, high stability and strong interactivity (Guo et al., 2016). Embedded technology provides a good platform for the design of human comfort meters. 
The traditional human comfort meter has the following defects: the interface is not uniform, the operation is cumbersome and complicated, the interface is unfriendly, and the stability and adaptability are poor. In order to effectively make up for the above defects, this paper newly designs a human comfort meter based on embedded technology. It uses the embedded development board to connect the temperature and humidity and wind speed sensors. The data is collected in real time through the LCD screen and gives the conclusion of comfort.

\section{Overall Design of Human Comfort Meter}

\subsection{Functional Requirements Analysis}

Preliminary design of a human comfort meter based on real-time operating system, using embedded development board and sensor to collect the temperature, relative humidity and wind speed data of the location in real time, and process the acquired data, and obtain the human body according to the basic algorithm model of human comfort. The comfort value is finally given according to the range of human comfort indicators. The temperature, humidity, wind speed, comfort value and conclusion result are displayed on the LCD screen. At the same time, the human comfort meter has data storage function and can expand the alarm function.

\subsection{System Design}

According to the functions that the human comfort meter needs to implement, the overall structural block diagram of the system is shown in Figure 1. The system is mainly composed of a core processor module, a sensor module, a signal acquisition and processing module, an RTC clock circuit, a debugging module, a data storage module and a display module, and can expand the alarm module.

The temperature and humidity sensor and the wind speed sensor collect temperature, relative humidity and wind speed data, and after processing by the signal processing circuit, finally enter the S3C2440 core processor module, and the processor applies the basic algorithm of the human comfort meter to process

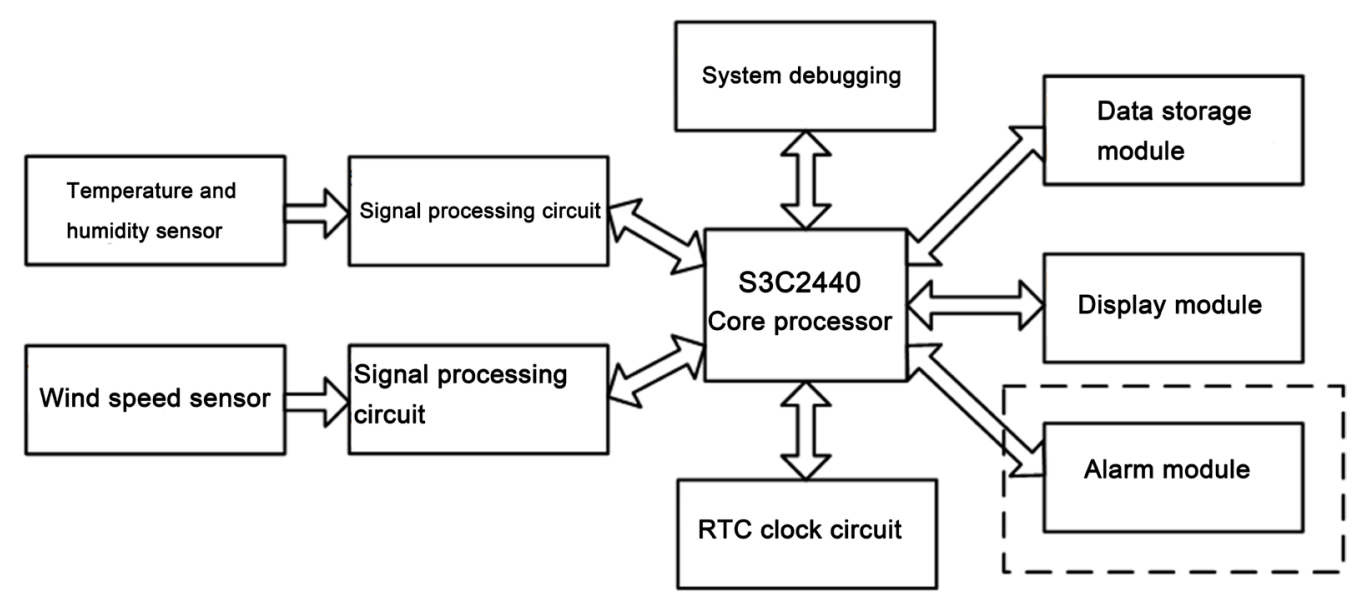

Figure 1. Overall structure of the system. 
the obtained data, and finally outputs the processing. After the temperature, relative humidity, wind speed, human comfort value and conclusion, the display program of the system is called to display. The RTC clock circuit is designed to provide accurate and reliable system time for the system to ensure the system has good real-time performance; the data storage module is used to save the data collected and processed by the system; the system debugging module is used for downloading and debugging the system program; the alarm module as an extended function, it can expand the sound and light alarm or increase the GSM module to send SMS alarms.

The main features of the human comfort meter designed in this paper are low power consumption, miniaturization, high precision measurement and rich expandability. In order to achieve the above features, the system uses Samsung's 32-bit RISC microprocessor S3C2440, which uses ARM920t core, has 0.13 micron COMS standard macro unit and memory unit and new bus architecture (Xia \& Niu, 2011); provides 1 channel LCD dedicated DMA, 4-channel PWM timer and 1-channel internal timer/watchdog timer, 8-channel 10-bit ADC and touch screen interface, 130 general purpose I/O ports and 24-channel external interrupt source, powered by $1.2 \mathrm{~V}$ core and $3.3 \mathrm{~V}$ external I/O supply, ideal for processing embedded applications that require high integration and low power consumption.

Embedded software is an integral part of embedded systems, including embedded operating systems and development tools. The embedded software system design mainly includes the following parts:

1) Initialization of the human comfort meter, preparation of the system startup code;

2) The migration of the embedded operating system, the kernel is tailored and configured according to the design requirements of the system;

3) The realization of the basic functions of the system, processing the data collected by the sensor and outputting;

4) Develop a graphical user interface using Qt Embedded and cross-compilation to display real-time data and human comfort conclusions.

\section{System Hardware Design}

\subsection{Human Comfort Meter Temperature and Humidity Acquisition Circuit Design}

The temperature and humidity measuring instruments commonly used in meteorological services include the HMP45D temperature and humidity sensor and the HMP155A temperature and humidity sensor produced by Vaisala, Finland. Although the performance is very good in terms of accuracy and stability, the price is high and the volume is large. Not suitable for use in this design. In this paper, the new digital temperature and humidity sensor SHT10 is used to obtain temperature and relative humidity data. The human body comfort meter temperature and humidity acquisition circuit are shown in Figure 2. 


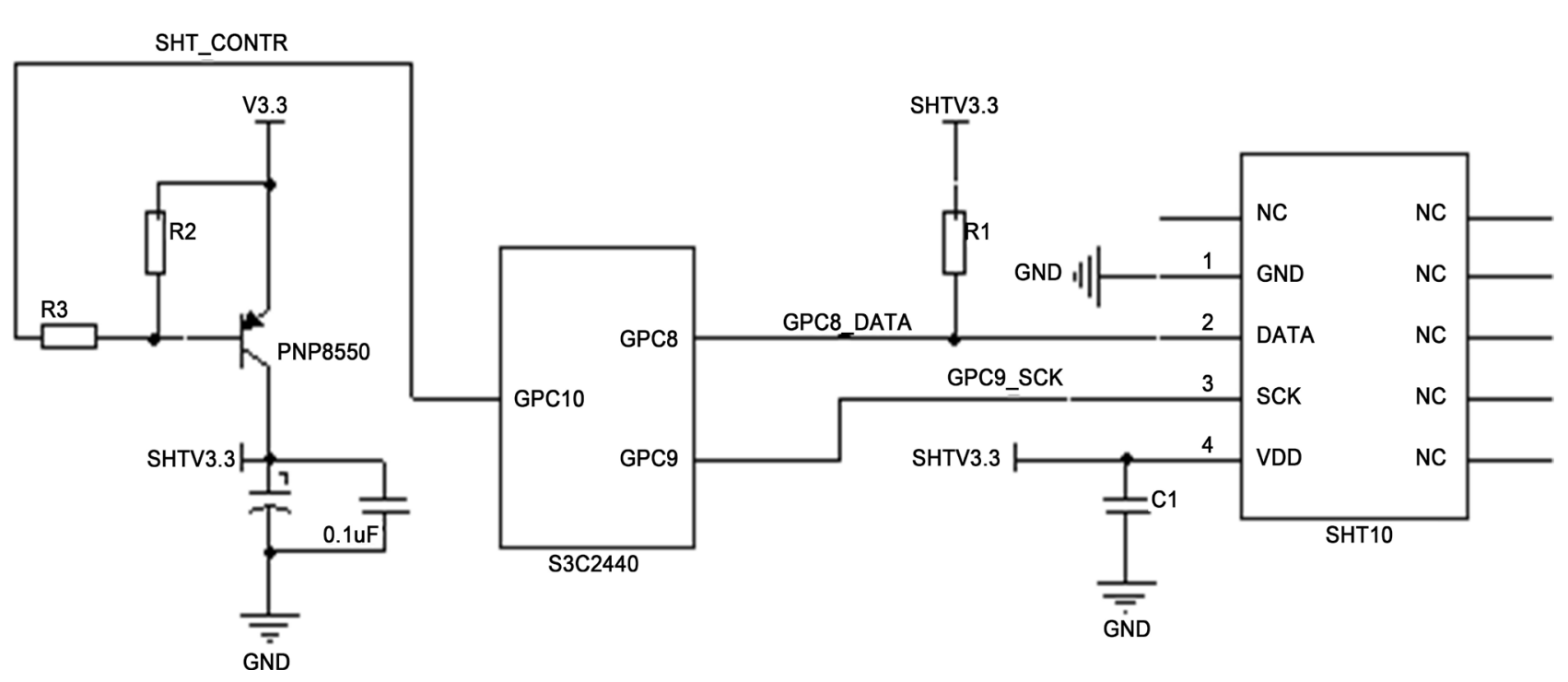

Figure 2. Human comfort meter temperature and humidity acquisition circuit.

The SHT10 is a temperature and humidity composite sensor with a calibrated digital signal output (Li \& Shi, 2011). It uses industrial CMOS process micromachining technology to make the sensor highly reliable and stable. The sensor consists of a bandgap temperature measuring element, a capacitive temperature measuring element, a 14-bit A/D converter and a serial interface circuit. The SHT10 sensor is accurately calibrated at the factory, and the calibration coefficient is stored in the OTP memory. In the process, the sensor needs to call the calibration coefficient (Ma et al., 2013) during the signal detection process. The sensor has excellent quality, ultra-fast response, strong anti-interference ability and high cost performance, which is very suitable for this design.

The SHT10 temperature and humidity sensor has a temperature resolution of $0.01^{\circ} \mathrm{C}$, a repeatability of $\pm 0.1^{\circ} \mathrm{C}$, a relative humidity resolution of $0.03 \%$ $R H$, and a repeatability of $\pm 0.1 \% R H$. As shown in Figure 2, the S3C2440 uses the GPC10 pin to control the SHT10 to reduce power consumption. The GPC8 and GPC9 pins are connected to the SHT10 DATA and SCK pins respectively. The SCK is used between the SHT10 and the S3C2440. Communication synchronization, DATA is used for data interaction between SHT10 and S3C2440. The S3C2440 first initializes the data transfer. It includes: When GPC9 outputs a high level, GPC8 outputs a low level, then GPC9 outputs a low level, and then GPC8 turns high when GPC9 outputs a high level. Thereafter, the S3C2440 sends a command to the SHT 10, the command byte is 8 bits, consisting of the upper 3 bits of the address bits and the lower 5 bits of the command bits, "00000011" for temperature measurement and "00000101" for relative humidity measurement. The S3C2440 indicates that the GPC8 is pulled low after the falling edge of the eighth SCK clock to indicate that the command was correctly received. After the falling edge of the ninth SCK clock, the S3C2440 releases the GPC8. 


\subsection{Human Comfort Meter Wind Speed Acquisition Circuit Design}

At present, there are roughly three types of sensors for measuring wind speed, namely a propeller type wind speed sensor, a three-cup type wind speed sensor, and an ultrasonic wind speed sensor. The measurement accuracy and measurement stability of the three-cup wind speed sensor are superior to the other two wind speed sensors. The design uses the three-cup wind speed sensor WM30 of Vaisala, Finland to measure the wind speed of the human comfort meter. The Vaisala Wind Sensor WM30 is a compact and economical wind speed and direction sensor (only wind speed measurement is used in this design). The rotating three-cup anemometer is located at the top of the entire sensor unit and provides a linear response to wind speed. The material of the cup the shape and size guarantee the accurate measurement of the wind speed, while the cup is rigorously tested to ensure a linear response between the wind speed and the angular speed of the cup wheel. Features and benefits of the WM30 include: lower cost, lighter and lighter design, the best choice for mobile applications, low power consumption, fast and linear response to wind.

The wind speed is output as a relay. The number of pulses in a fixed time can be recorded to calculate the wind speed. The wind speed can also be calculated by measuring the time interval between successive pulses. In this paper, the number of pulses in a fixed time is recorded to calculate the wind speed. The human body comfort meter wind speed acquisition circuit is shown in Figure 3.

In Figure 3, P1 is a transfer port for connecting the wind speed sensor WM30, wherein the pin 3 is a signal output port; the TLP521 is an optocoupler element for isolating the wind speed sensor from the S3C2440 to reduce signal crosstalk, the TLP521 pin 1 is the signal input terminal, connected to pin 3 of P1, pin 3 of TLP521 is the signal output port, and two non-gate devices are connected in series and connected to the GPD0 port of S3C2440. The wind speed sensor signal output calculation formula is shown in Formula (1).

$$
V=-0.24+0.699 * F
$$

where $V$ is the wind speed (in $\mathrm{m} / \mathrm{s}$ ) and $F$ is the pulse frequency (in $\mathrm{Hz}$ ) of the

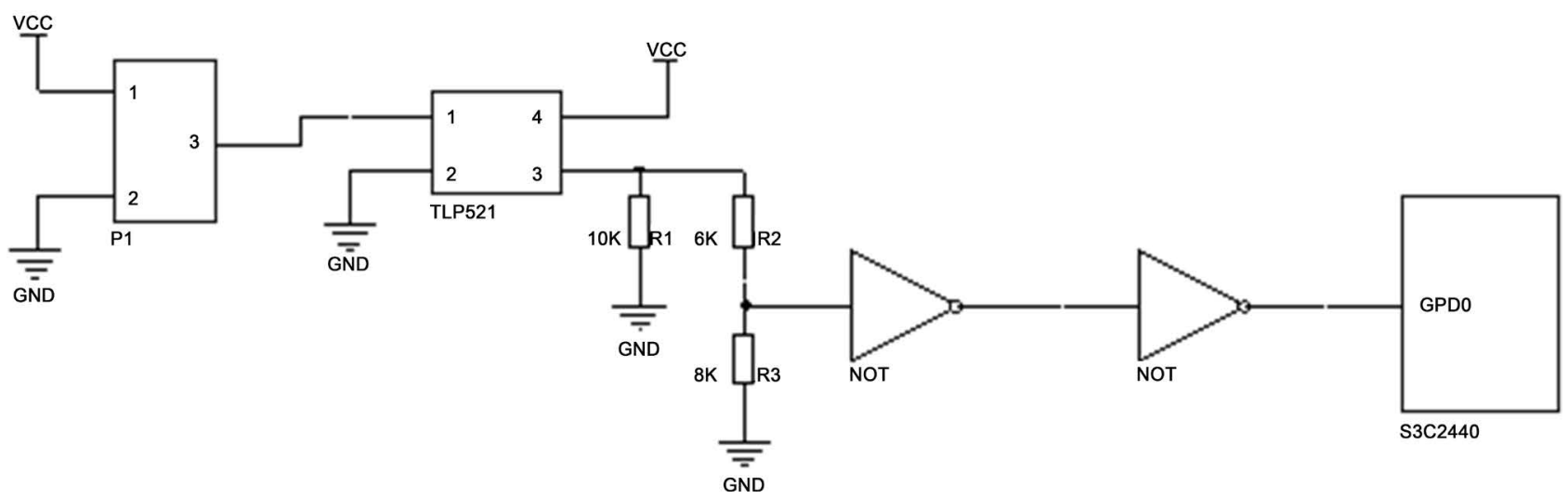

Figure 3. Human comfort meter wind speed acquisition circuit. 
wind speed sensor output.

\section{System Software Design}

\subsection{Software Design Environment}

In an embedded system, the source file code must be compiled to be converted into an executable binary object file (Zhang et al., 2012). A complete embedded system requires a lot of object files, which requires a link tool to link all the target files into a total binary object file. The tools involved include compilers, linkers, relocatable programs, and locators.

In this design, the host environment needs to be built. The solution is to install the virtual machine on the PC, and then install the Linux operating system in the virtual machine environment. The host and the target communicate with each other through the network. The IP address of the Linux operating system of the virtual machine needs to be in the same network segment as the IP of the Windows operating system. In order to generate executable code on another platform on one platform, you need to build a cross-compilation environment and install cross-compilation tools.

The transplantation of embedded operating system is an important step in the process of building software development environment. The operating system ported in this design is Linux, which mainly includes the transplantation of system bootloader, kernel porting, and root file system porting.

\subsection{System Software Design}

The system software design includes system driver design, Qt/Embedded graphical user interface programming and human comfort instrument algorithm program.

System drivers include device drivers, temperature and humidity sensor drivers, and wind speed sensor drivers. The application communicates with the kernel via a Linux system call. Both the temperature and humidity sensors and the wind speed sensor are connected to the I/O port of the S3C2440, while the I/O device is used through a fixed entry point defined by the device driver. The driver of the temperature and humidity sensor SHT10 is calculated by changing the output value of the GPC9 port of the S3C2440 and reading the input value of the GPC8 port according to the working principle of the SHT10. The working principle of the specific code has been described in detail above. Let me repeat. The driver of the wind speed sensor is relatively simple, mainly to enable a timer to record the number of pulses of the GPDO port in a fixed time, and calculate by the Formula (1).

Qt is a cross-platform $\mathrm{C}++$ graphical user program that provides signal and slot object communication mechanisms with queryable and designable properties, as well as powerful event and event filters (Jin \& Cui, 2014). Qt/Embedded is a complete GUI and Linux-based embedded platform development tool. Using $\mathrm{Qt} /$ Embedded to write a graphical user program is to apply its good cross-platform 
properties, that is, to write programs and debug on the Windows platform, and finally Run on an embedded platform. In this design, Qt/Embedded interacts with the S3C2440's I/O facilities through the Qt API. The construction of the Qt environment includes the installation of the Qt Creator software and the construction of the cross-compilation environment under the Linux platform. Using Qt to develop the application program of human comfort meter, the program interface has temperature, relative humidity, wind speed, comfort value and comfort conclusion display function. After the program is compiled, the executable file is generated, and the executable file is downloaded to the S3C2440 development board. The design of the Qt/Embedded graphical user interface program is completed.

The human comfort tester algorithm uses the human comfort index prediction model used by the Beijing Meteorological Administration since 1997. The nonlinear equation of the model is shown in Equation (2).

$$
D I=1.8 T+0.55(1-R H)+32-3.2 \sqrt{V}
$$

In the formula, $D I$ is the calculated comfort value, $T$ is the temperature, $R H$ is the relative humidity, and $V$ is the wind speed. The higher the $D I$ value, the hotter the human body feels, and the lower the $D I$ value, the colder the human body feels. The correspondence between the $D I$ value and the conclusion of human comfort is shown in Table 1 . The rank of $D I$ calculated values is determine by the Beijing Meteorological Administration of China.

It can be seen from Table 1 that when the calculated value of $D I$ is too large or too small, the human body feels uncomfortable. Only when the $D I$ value is moderate, that is, the temperature, relative humidity, and wind speed are controlled within a certain range. The feeling is more comfortable.

\section{Conclusion}

This paper designs a human comfort meter based on embedded processor and

Table 1. Correspondence between $D I$ calculated values and human comfort conclusions.

\begin{tabular}{ccc}
\hline Serial number & $D I$ calculated value & Comfort conclusion \\
\hline 1 & $D I<0$ & very cold and uncomfortable \\
2 & $0 \leq D I \leq 25$ & very cold, uncomfortable \\
3 & $26 \leq D I \leq 38$ & cold, uncomfortable \\
4 & $39 \leq D I \leq 50$ & a few people comfortable \\
5 & $51 \leq D I \leq 58$ & most people are comfortable \\
6 & $59 \leq D I \leq 70$ & comfortable \\
7 & $71 \leq D I \leq 75$ & warm, most people are comfortable \\
8 & $76 \leq D I \leq 79$ & hot, a few people are not comfortable \\
9 & $80 \leq D I \leq 84$ & hot, most people are not comfortable \\
10 & $85 \leq D I \leq 88$ & hot, uncomfortable \\
11 & $D I>88$ & very hot, very uncomfortable \\
\hline
\end{tabular}


operating system. By collecting the temperature, relative humidity and wind speed data in the environment, combined with the human comfort algorithm model, the conclusion of human comfort is obtained. The software development environment, through Qt to write a graphical user interface to display the measurement values, comfort values and comfort conclusions of each element. After testing, the human comfort meter can accurately collect the values of various elements in the environment, and the real-time performance is good. The system software and hardware design are reasonable; the function is perfect; the reliability is high, meets the expected design goals, and has a good application prospect.

The human comfort index is a bio-meteorological indicator based on the meteorological point of view to evaluate the comfort of people in different climatic conditions and based on the heat exchange between the human body and the atmospheric environment. In general, the three meteorological elements of temperature, relative humidity and wind speed have the greatest impact on human body perception. The application of this product can help people understand the atmospheric environment, take timely measures to prevent people from happening, and reduce the mistakes in work and life decisions caused by emotions.

\section{Acknowledgements}

None.

\section{Conflicts of Interest}

The authors declare no conflicts of interest regarding the publication of this paper.

\section{References}

Chen, L., Wu, Z., \& Liu, Y. (2013). Porting of Embedded Operating System on TMS320F28335 Platform. Computer Technology and Development, 23, 7-11.

Guo, S., Feng, H., \& Zhou, S. (2016). Design of Portable Instrument Using Microcontroller Operating System. Automation Instrumentation, 37, 93-95.

Jin, F., \& Cui, P. (2014). Research on Signal and Slot Mechanism in Embedded Qt. Electronic Design Engineering, 22, 168-170.

Kai, Z., Kan, Z., \& Zhang, F. (2014). Evaluating Bus Transit Performance of Chinese Cities: Developing an Overall Bus Comfort Model. Transportation Research Part A: Policy \& Practice, 69, 105-112. https://doi.org/10.1016/j.tra.2014.08.020

Li, J., Wang, Y., Peng, N. et al. (2016). Analysis of Tourism Meteorological Conditions in Shilin County. Anhui Agricultural Sciences, 44, 200-201.

Li, L., \& Shi, W. (2011). Design and Implementation of Intelligent Temperature and Humidity Control System in Greenhouses. Hunan Agricultural Sciences, 21, 135-138.

Ma, D., Wang, W., Jiang, Q. et al. (2013). On-Line Temperature and Humidity Monitoring System Based on RS485 Bus. Chinese Journal of Agricultural Mechanization, 34, 121-126.

Mostafavi Tehrani, S. S., Taylor, R. A., Nithyanandam, K., \& Shafiei Ghazani, A. (2017). 
Annual Comparative Performance and Cost Analysis of High Temperature, Sensible Thermal Energy Storage Systems Integrated with a Concentrated Solar Power Plant. Solar Energy, 153, 153-172. https://doi.org/10.1016/j.solener.2017.05.044

Moustris, K., Tsiros, I. X., Tseliou, A., \& Nastos, P. (2018). Development and Application of Artificial Neural Network Models to Estimate Values of a Complex Human Thermal Comfort Index Associated with Urban Heat and Cool Island Patterns Using Air Temperature Data from a Standard Meteorological Station. International Journal of Biometeorology, 4, 1-10. https://doi.org/10.1007/s00484-018-1531-5

Peng, J., Zong, Z., Huang, X. et al. (2011). Evaluation of Climate Comfort and Prediction Equation for Rafting in Mengdong River, Hunan Province. Meteorology, 37, 771-776.

Slater, L. J., Villarini, G., \& Bradley, A. A. (2017). Evaluation of the Skill of North-American Multi-Model Ensemble (NMME) Global Climate Models in Predicting Average and Extreme Precipitation and Temperature over the Continental USA. Climate Dynamics, 46, 1-16. https://doi.org/10.1007/s00382-016-3286-1

Takaya, Y., Yasuda, T., Fujii, Y., Matsumoto, S., Soga, T., Mori, H. et al. (2017). Japan Meteorological Agency/Meteorological Research Institute-Coupled Prediction System Version 1 (jma/mri-cps1) for Operational Seasonal Forecasting. Climate Dynamics, 48, 313-333. https://doi.org/10.1007/s00382-016-3076-9

Wallace, Z. C., \& Hill, A. A. (2017). Forecaster and Emergency Manager Perspectives on Coordination and Communication with the Weather-Warned Public. Papers in Applied Geography, 3, 157-170. https://doi.org/10.1080/23754931.2017.1299036

Wang, B., Bai, X., Zhang, C. et al. (2015). Embedded Software Test Case Generation Based on Interface Automata and Symbolic Execution. Chinese Journal of Computers, $38,2125-2144$.

Xia, J., \& Niu, C. (2011). Research and Implementation of Linux Porting Based on S3C2440A Processor. Computer and Digital Engineering, 39, 77-80.

Zhang, H., Gao, L., \& Song, C. (2012). Research and Implementation of Embedded Linux Cross-Compilation Environment Based on ARM. Computer and Digital Engineering, 40, 151-153.

Zhu, M., Yin, X., Qu, X. et al. (2014). The Temporal and Spatial Distribution Trend of Summer Human Body Comfort Index in Hulunbeier City. Inner Mongolia Agricultural Science and Technology, 3, 94-96. 